\title{
Significance of Linked Color Imaging for Predicting the Risk of Clinical Relapse in Ulcerative Colitis
}

\author{
Shuji Kanmura (D), Akihito Tanaka, Kazuki Yutsudou, Kosuke Kuwazuru, Fukiko Komaki, \\ Yuga Komaki, Hiromichi Iwaya, Shiho Arima, Fumisato Sasaki, Shiroh Tanoue, \\ Shinichi Hashimoto $(\mathbb{D}$, and Akio Ido
}

Digestive and Lifestyle Diseases, Kagoshima University Graduate School of Medical and Dental Sciences, Kagoshima, Japan

Correspondence should be addressed to Shuji Kanmura; skanmura@m2.kufm.kagoshima-u.ac.jp

Received 26 November 2019; Revised 15 February 2020; Accepted 24 February 2020; Published 6 March 2020

Academic Editor: Agata Mulak

Copyright ( $\odot 2020$ Shuji Kanmura et al. This is an open access article distributed under the Creative Commons Attribution License, which permits unrestricted use, distribution, and reproduction in any medium, provided the original work is properly cited.

\begin{abstract}
Ulcerative colitis (UC) is a chronic inflammatory bowel disease with unknown etiology. Recently, mucosal healing has emerged as an important therapeutic endpoint in UC. Linked color imaging (LCI) is a novel endoscopic system that enhances the color differences of the gastrointestinal mucosa. Our previous study emphasized the redness and yellowness of the lesion using LCI observation, which was useful for the evaluation of histological mucosal activity in UC. In this study, we aimed to evaluate the correlation between LCI observation and clinical relapse rate in UC patients. We retrospectively analyzed UC patients who underwent total colonoscopy between August 2016 and October 2018 at our facility with Mayo endoscopic scores of 0 or 1 . We assessed the correlation between orange-like color lesion (defined as LCI-scarlet color lesions) and clinical relapse rate (requiring additional treatment for UC) during the 1-year follow-up period. Fifty-eight patients (22 female, 36 male; median age at diagnosis, 47.2 (18-80) years) who underwent colonoscopy were analyzed. During the 1-year follow-up period, clinical relapse was observed in 12 patients (20.1\%) among which ten patients (83.3\%) had an LCI-scarlet color lesions recognized by LCI. By contrast, 29 patients $(63 \%)$ had no LCI-scarlet color lesions in the clinical remission group $(n=46)$. There was a significant difference in LCI-scarlet color between the clinical relapse and remission groups, remaining significantly associated with clinical relapse. LCI findings, including an orange-like color lesion, have diagnostic implications for predicting the risk of clinical relapse in UC during the 1-year follow-up period.
\end{abstract}

\section{Introduction}

Ulcerative colitis (UC) is a chronic inflammatory bowel disease of unknown etiology. Colonoscopy and biopsy have been established as the methods of choice to diagnose UC [1]. Recently, mucosal healing $(\mathrm{MH})$ has emerged as an important therapeutic endpoint in UC [2-4]. MH has been reported to reduce the rates of disease relapse, hospital admission, and surgery, as well as lower the cumulative risk of dysplasia and colorectal cancer progression [5-8]. Endoscopic MH has been defined as a Mayo endoscopic scores (MES) of 0 or 1 using conventional white light imaging (WLI) in most clinical trials [9-12]. MH is usually diagnosed and confirmed based on endoscopic observation using WLI, as reported in a recent study [13].
Linked color imaging (LCI), a color enhancement function of the LASEREO system, has been developed as a new image-enhanced endoscopy (IEE) technology. This system makes red areas appear redder and white areas appear brighter. Therefore, it is a useful tool for recognizing color differences in the mucosa, by facilitating the detection and recognition of colorectal neoplasms [14-19].

In addition, the utility of LCI in the assessment of histological intestinal inflammation in UC patients has been reported $[20,21]$. In our previous study, findings of endoscopic observations by LCI-a and LCI-b, which emphasized redness and yellowness of lesion, showed a significant difference between mucosal inflammation and noninflammation. Therefore, mixed reddish and yellowish color site (an orange-like color) using LCI observation might be 
useful for detecting and visualizing mucosal inflammation. In this study, we aimed to evaluate the association between endoscopic assessment using LCI observation and clinical relapse rate in UC patients.

\section{Materials and Methods}

2.1. Patients. We retrospectively enrolled UC patients who underwent endoscopy between August 2016 and October 2018 at Kagoshima University Hospital. All patients were diagnosed with UC using established endoscopic, histological, and clinical criteria. Endoscopic evaluation of disease inflammatory activity was performed using MES, defined as MES 0, a normal mucosa or inactive disease; MES 1, mild activity (erythema, decreased vascular pattern, and mild friability); MES 2, moderate activity (marked erythema, lack of vascular pattern, friability, and erosions); or MES 3, severe activity (spontaneous bleeding and large ulcerations). UC patients in sustained corticosteroid-free remission $(\geq 6$ months) underwent total colonoscopy, and patients with MES 0 or MES 1 were included in the study. Patients with MES 2 or 3 or a history of total colectomy, colitic cancer, or unclassified inflammatory bowel disease were excluded. Clinical remission was defined as rectal bleeding subscores of 0 (no rectal bleeding) and stool frequency subscores of either 0 (normal stool frequency for the patient) or 1 ( 1 or 2 more daily stools than normal) using the partial Mayo scoring system. The rate of clinical relapse was investigated during the 1-year follow-up period. The observation started 1 year from the date of colonoscopy. The clinical relapse was defined during this period as the need for intensification or modification of medication, or UCrelated hospital admission, or surgery.

2.2. Endoscopic Procedure. Routine bowel preparation was done by Moviprep ${ }^{\circledR}$ that contained polyethylene glycol 3350 , sodium sulfate, sodium chloride, potassium chloride, sodium ascorbate, and ascorbic acid. Conventional colonoscopy was performed by an EC-L600ZW endoscope with the LASEREO system that consisted of a VP-4450HD processor and an LL-4450 light source (Fujifilm Co., Tokyo, Japan) and could produce light suitable for WLI and LCI. Two experienced endoscopists evaluated the MES using WLI and LCI endoscopic findings in each case. The endoscopic factors, including marked erythema, vascular pattern, and friability, were evaluated based on both LCI and WLI observations. The orange-like color site, emphasizing redness and yellowness by LCI observation, was defined as LCI-scarlet color lesion.

2.3. Assessment of Clinical Outcome. The primary endpoint was to determine the association between endoscopic findings, including LCI-scarlet color lesion and risk of clinical relapse in UC patients. The secondary endpoint was to assess predictive factor among clinical parameters for the risk of clinical relapse, which included age, gender, duration of disease, extent of disease, disease type (first attack, relapsing/remission type, or chronic continuous type), and concomitant medications at the time of colonoscopy procedure. The pres- ent study was approved by the Kagoshima University Hospital Institutional Review Board and performed in accordance with the Declaration of Helsinki. Written informed consent was obtained from all patients.

2.4. Statistical Analyses. Results were analyzed using the Mann-Whitney $U$ test or Wilcoxon signed-rank test, as appropriate. Correlation coefficients were calculated by Spearman's rank correlation analysis. Univariate analyses were performed using the $\chi^{2}$ test for categorical variables and multivariate analysis using a logistic regression model with the calculation of odds ratios and 95\% confidence intervals (CI). Kaplan-Meier curves for the duration of clinical remission during follow-up were generated for UC patients and were compared using the 2-side log-rank test. Kappa values were calculated for the interobserver agreement between two endoscopists to validate the MES and LCIscarlet color. All statistical analyses were conducted using the SPSS software program (version 22; SPSS Inc., Chicago, IL, USA). $p$ values $<0.05$ were considered statistically significant.

\section{Results}

3.1. Patient Clinical Characteristics. We assessed 94 UC patients who underwent endoscopy during the study period. Of these, 36 were excluded based on our exclusion criteria (colitic cancer, $n=3$; inflammatory bowel disease unclassified, $n=2$; MES $\geq 2, n=19$; endoscopic observation <1-year, $n=12$ ), and 58 were analyzed (male, 36, 62\%); median age, 47.2 years). During the 12 -month follow-up period, clinical relapse was observed in 12 patients (20.1\%; Figure 1$)$. The median relapse time from the start of the observation period was 6.7 months (range, 2.5-11.2 months).

For endoscopic inflammatory activity, MES 0 was observed in 22 (38\%), and MES 1 was observed in $36(62 \%)$ patients. The kappa value for MES was very good (kappa $=0.70)$. Thirty-three patients were treated with only aminosalicylates, eight with aminosalicylates and azathioprine, six with aminosalicylates and antitumor necrosis factor alpha (TNF $\alpha$ ) agents (infliximab and adalimumab), and ten with aminosalicylates, azathioprine, and $\mathrm{TNF} \alpha$ agents. One patient had no medication. Table 1 summarizes the baseline characteristics of patients. Among these 12 patients, intensification of the current therapy was needed in seven, corticosteroids were started in two, and an anti-TNF $\alpha$ drug in three. The rate of clinical relapse at 1-year follow-up (requiring additional treatment for UC) showed no significant difference between MES 0 and MES 1 patient groups based on WLI observation, although the rate in patients with MES 0 group tended to be lower than that in the MES 1 group (Figure 2).

\subsection{LCI-Scarlet Color Predicting Prognosis in UC Patients} with Clinical Remission. The LCI-scarlet color was evaluated at the site that appeared to have high activity. A representative case is shown in Figure 3. The kappa value for LCIscarlet color was very good (kappa value $=0.89$ ). 


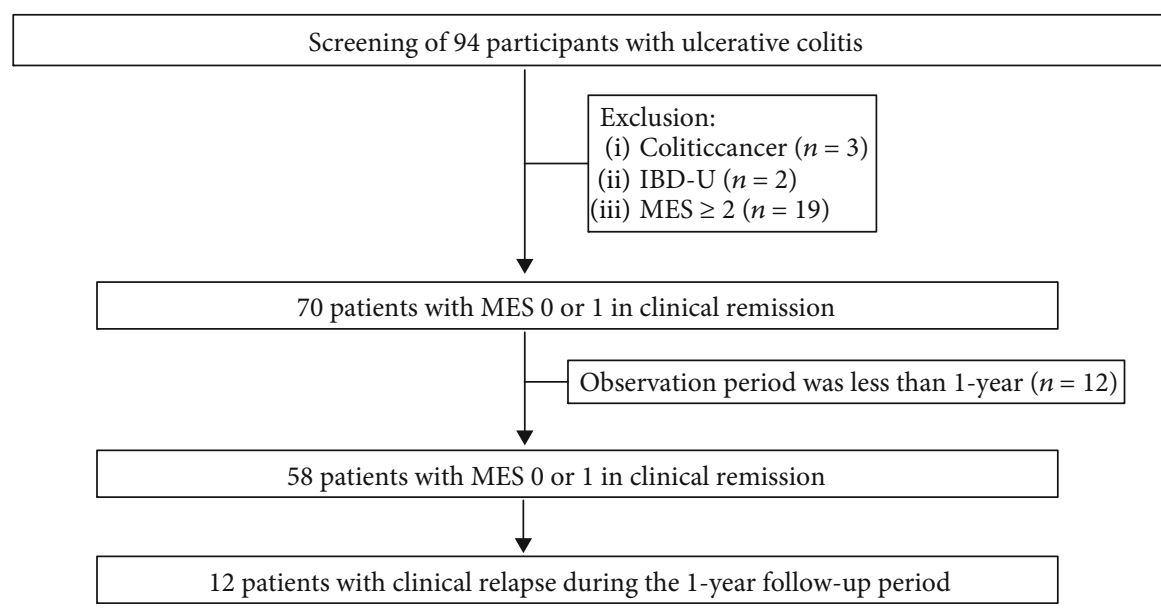

FIGURE 1: Study flowchart summarizing the selection of ulcerative colitis patients. Abbreviations: IBD-U: inflammatory bowel disease unclassified; MES: Mayo endoscopic score.

TABLE 1: Clinical characteristics of the patients with ulcerative colitis.

\begin{tabular}{lc}
\hline Number of patients & 58 \\
\hline Gender & 36 \\
Male & 22 \\
Female & $47.2(18-80)$ \\
Median age (range), year & \\
Mayo endoscopic score & 22 \\
0 & 36 \\
1 & \\
Extent of disease & 6 \\
Rectum & 13 \\
Left-sided colitis & 39 \\
Pancolitis & \\
Current treatment & 1 \\
None & 33 \\
5-ASA only & 8 \\
5-ASA and azathioprine & 6 \\
5-ASA and TNF $\alpha$ agents & 10 \\
5-ASA and azathioprine and TNF $\alpha$ agents
\end{tabular}

List of abbreviations: 5-ASA: 5-aminosalicylic acid; TNF $\alpha$ : antitumor necrosis factor-alpha.

Clinical relapse was observed in 12 patients, among whom ten (83.3\%) had an LCI-scarlet color lesion recognized by LCI. By contrast, 29 patients (63\%) had no LCI-scarlet color lesions in the clinical remission group $(n=46)$. Twenty-seven patients showed LCI-scarlet color lesions of which ten had clinical relapses (37.0\%). By contrast, among patients with no lesions $(n=31)$, clinical relapse was observed in two patients $(6.5 \%)$.

There were two cases $(9.1 \%)$ positive for LCI-scarlet in MES 0 and 25 cases (69.4\%) positive for LCI-scarlet in MES1. The relapse rate of UC of LCI-scarlet-positive patients in MES0 was $100 \%(2 / 2)$; those in MES1 was $40 \%(10 / 25)$.

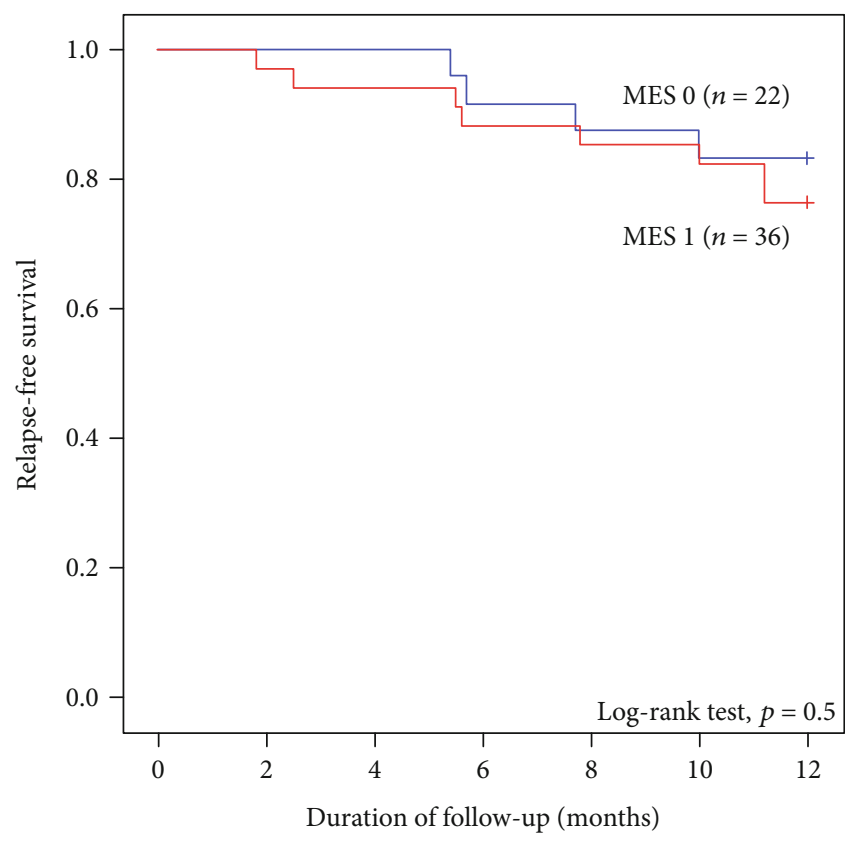

FIgURE 2: Kaplan-Meier curves of relapse-free survival for total ulcerative colitis (UC) patients with Mayo endoscopic scores 0 and $1(n=58)$ using the log-rank test. Clinical relapse was defined as additional or changed treatment for UC. Abbreviation: MES: Mayo endoscopic score.

Figure 4 shows the Kaplan-Meier curves of relapse-free survival for patients based on LCI-scarlet color. The relapse rate in patients with LCI-scarlet color was significantly higher than that in patients without LCI-scarlet color (logrank test, $p=0.02$ ).

3.3. Correlation of LCI Findings with the Risk of Clinical Relapse in UC Patients. The duration of UC was significantly shorter in patients with clinical relapse than those with remission (average year, 7.4 vs $13.4 ; p=0.047$ ). Assessment of the association of LCI-scarlet color lesion with the risk of 


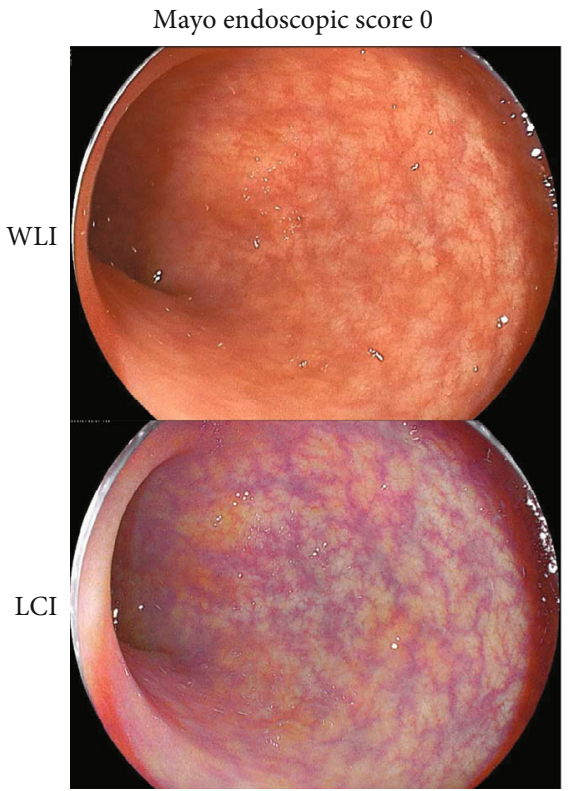

Negative for LCI-scarlet
Mayo endoscopic score 0

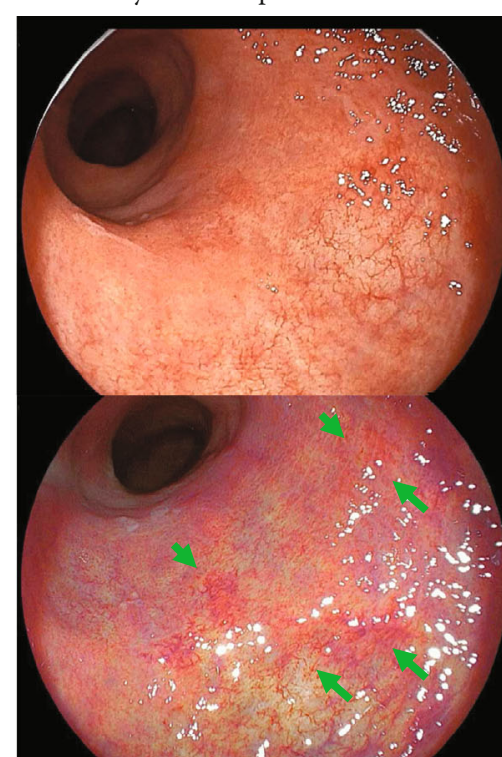

Positive for LCI-scarlet

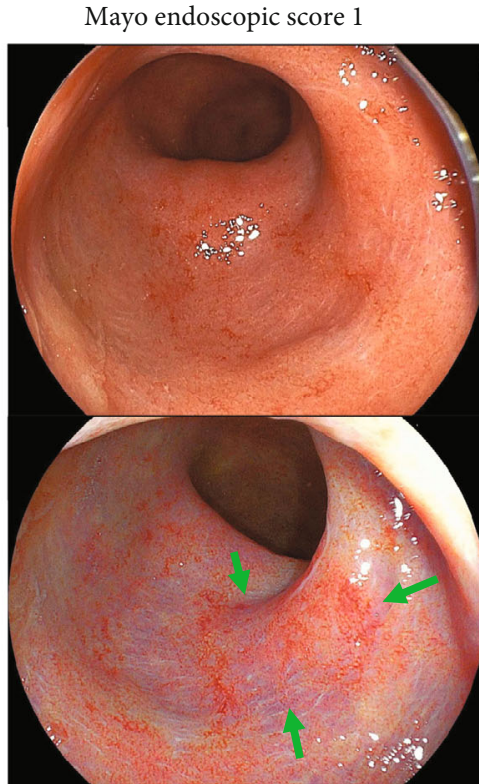

Positive for LCI-scarlet

FIGURE 3: Endoscopic images of the colon segments obtained from a representative ulcerative colitis case with Mayo endoscopic score 0 and 1 observed under linked color imaging (LCI) and white light imaging (WLI). Lower panel shows the LCI-scarlet color assessment. Arrows indicate the LCI-scarlet color area.

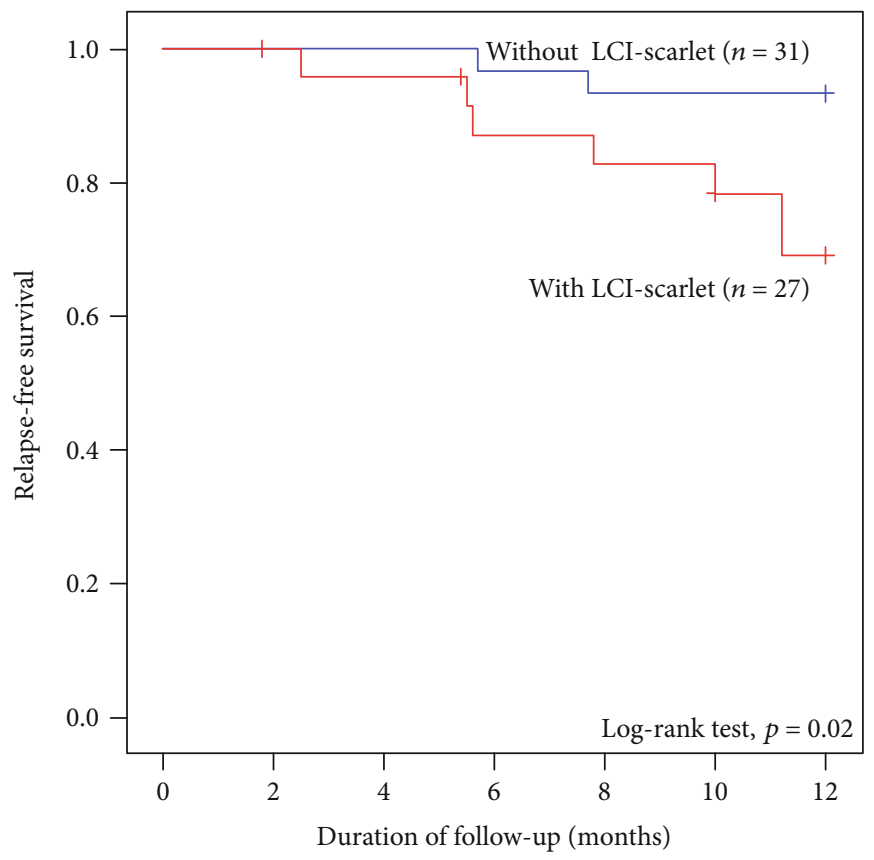

FIGURE 4: Kaplan-Meier curves of relapse-free survival for ulcerative colitis patients with or without linked color imaging (LCI)-scarlet color site using the log-rank test. Abbreviation: LCI, linked color image.

clinical relapse revealed the relapse rate of patients with LCI-scarlet color to be significantly higher than those without LCI-scarlet color $(37.0 \%$ vs $3.2 \%$; $p=0.002)$. Other clinical parameters, including gender, age, extent of disease, and current concomitant medications (5-aminosalicylic acid, azathioprine, and TNF $\alpha$ ) showed no correlation with disease relapse. In multivariate analysis, LCI-scarlet color was the only independent factor that showed a significant association with clinical relapse (odds ratio, $14.8 ; 95 \% \mathrm{CI}$, $1.68-130.4 ; p<0.015)$.

\section{Discussion}

We investigated the correlation between endoscopic findings based on LCI and the risk of clinical relapse in patients with UC. The relative findings included a significant association of 
the scarlet color lesion under LCI observation with clinical relapse in UC patients, based on multivariable analysis. The LCI-scarlet color lesion may suggest mucosal inflammation, as reported in our previous study [21].

$\mathrm{MH}$ has emerged as an important therapeutic goal for UC patients. The follow-up study of the Active UC trials 1 and 2 reported that $\mathrm{MH}$ after 8 weeks of infliximab treatment correlated with improved clinical outcomes [8]. Although previous clinical studies defined MH as an MES of 0 or 1, it was reported that patients with MES 1 had significantly higher long-term risk of clinical relapse than those with MES 0; therefore, the concept of $\mathrm{MH}$ should be limited to patients with MES of $0[8,13,22,23]$. A high rate of disagreement in endoscopic scoring was found in MES even among experienced physicians [24]. Although MES is the most commonly used method, its application as MES 0 or 1 in a complicated case remains controversial for the reasons mentioned above. Therefore, a more accurate and simple endoscopic observation is required. Based on our findings, we suggest the utility of LCI-scarlet color sign for the assessment of $\mathrm{MH}$.

Various IEE modalities, including narrow-band imaging (NBI), blue-laser imaging, and LCI, have been developed, among which NBI and LCI are efficacious in evaluating the severity of histological inflammation in UC [20, 21, 25-27]. IEE has been frequently used in clinical practices, including detection and characterization of sporadic colonic tumor [14-19, 28-31], detection of tumor lesion, and diagnosis of histological activity, and has been recently suggested for predicting relapse risk $[20,26]$. We previously reported that LCI was more useful than conventional endoscopy for the visualization and evaluation of mucosal inflammation based on the mucosal color change of redness and yellowness in UC. LCI color value correlated with histological mucosal inflammation score [21]. LCI observation emphasizes minute differences in colors, resulting in a more accurate mucosal inflammation. In the present study, LCI observations were useful for assessing the clinical outcome of UC by focusing on the color differentiation. We also reported the utility of LCI-scarlet color lesion for the prediction of clinical relapse in UC. In line with our study, Uchiyama et al. reported correlations of colonic mucosal redness enhanced by LCI with mucosal inflammation. They classified endoscopic images under LCI observation based on the degree of mucosal redness and visible vessels and suggested this LCI classification for predicting UC relapse [20]. These findings suggest that LCI would be more useful than WLI for the diagnosis of inflammatory UC.

Endoscopic evaluation of disease inflammatory activity based on MES showed no significant difference in clinical relapse rate between MES 0 and 1 patient groups; however, a trend for higher relapse rate in the patients with MES 1 was noted in this study. By contrast, the LCI finding was suggested for predicting the risk of clinical relapse in UC patients, implicating its role as a novel diagnostic approach for evaluating the colonic mucosa in UC patients for assessing $\mathrm{MH}$ accurately.

This study has several limitations. First, there was a single-center retrospective study design; therefore, it is neces- sary to consider a large-scale prospective study for assessing the diagnostic utility of LCI in UC. Second, two endoscopists evaluated the endoscopic findings using the images obtained by WLI or LCI in all cases rather than analyzing while performing the endoscopic procedure. Finally, we investigated clinical relapse based only on the requirement of additional treatment for UC as the outcome. Although we planned to consider hospitalization and surgical rates, however, due to low hospital admission rate $(0.03 \%, 2 / 58$ cases $)$ and no surgical requirement $(0 \%, 0 / 58$ cases) during the follow-up period, we failed to assess the correlation of these rates with LCI observation.

\section{Conclusions}

For assessment of MH, LCI may be defined as no scarlet color lesion in patients with MES 0 or 1 . Endoscopic findings by LCI predict the risk of clinical relapse in UC patients and are likely to aid clinicians.

\section{Data Availability}

The data used to support the findings of this study are included within the article. Additional data are available from the corresponding author (Shuji Kanmura, skanmur$\mathrm{a} @ m 2 . k u f m . k a g o s h i m a-u . a c . j p)$ for researchers who meet the criteria for access to confidential data.

\section{Conflicts of Interest}

The authors declare that there is no conflict of interest regarding the publication of this article.

\section{Authors' Contributions}

SK and AI did the concept and study design. SK, AT, KY, KK, FK, YK, HI, SA, FS, ST, and SH did the medical examination and colonoscopy performance. SK did the data analysis. SK and AT did the analysis using statistical tools. SK and AI drafted the manuscript.

\section{Acknowledgments}

We thank Dr. Yuichi Teramura, FUJIFILM for his valuable technical advice, and we would like to thank Editage (https://www.editage.com) for English language editing. SK, AI: concept and study design. SK, AT, KY, KK, FK, YK, HI, SA, FS, ST, SH: medical examination and colonoscopy performance. SK: data analysis. SK, AT: analysis using statistical tools. SK, AI: drafting of the manuscript.

\section{References}

[1] R. Ungaro, S. Mehandru, P. B. Allen, L. Peyrin-Biroulet, and J.-F. Colombel, "Ulcerative colitis," The Lancet, vol. 389, no. 10080, pp. 1756-1770, 2017.

[2] J. B. Chevaux, S. R. Vavricka, G. Rogler, P. L. Lakatos, A. Schoepfer, and L. Peyrin-Biroulet, "Mucosal healing with anti-TNF antibodies," Digestion, vol. 86, Supplement 1, pp. 16-22, 2012. 
[3] G. Pineton de Chambrun, L. Peyrin-Biroulet, M. Lémann, and J. F. Colombel, "Clinical implications of mucosal healing for the management of IBD," Nature Reviews Gastroenterology \& Hepatology, vol. 7, no. 1, pp. 15-29, 2010.

[4] G. Fiorino, M. Cesarini, A. Indriolo, and A. Malesci, "Mucosal healing in ulcerative colitis: where do we stand?," Current Drug Targets, vol. 12, no. 10, pp. 1417-1423, 2011.

[5] F. Carbonnel, A. Lavergne, M. Lémann et al., "Colonoscopy of acute colitis. A safe and reliable tool for assessment of severity," Digestive Diseases and Sciences, vol. 39, no. 7, pp. 1550-1557, 1994.

[6] K. F. Frøslie, J. Jahnsen, B. A. Moum, M. H. Vatn, and IBSEN Group, "Mucosal healing in inflammatory bowel disease: results from a Norwegian population-based cohort," Gastroenterology, vol. 133, no. 2, pp. 412-422, 2007.

[7] J. F. Colombel, P. Rutgeerts, W. Reinisch et al., "Early mucosal healing with infliximab is associated with improved long-term clinical outcomes in ulcerative colitis," Gastroenterology, vol. 141, no. 4, pp. 1194-1201, 2011.

[8] F. Casellas, M. Barreiro de Acosta, M. Iglesias et al., "Mucosal healing restores normal health and quality of life in patients with inflammatory bowel disease," European Journal of Gastroenterology \& Hepatology, vol. 24, no. 7, pp. 762769, 2012.

[9] G. D'Haens, W. J. Sandborn, B. G. Feagan et al., “A review of activity indices and efficacy end points for clinical trials of medical therapy in adults with ulcerative colitis," Gastroenterology, vol. 132, no. 2, pp. 763-786, 2007.

[10] K. W. Schroeder, W. J. Tremaine, and D. M. Ilstrup, “Coated oral 5-aminosalicylic acid therapy for mildly to moderately active ulcerative colitis. A randomized study," The New England Journal of Medicine, vol. 317, no. 26, pp. 1625-1629, 1987.

[11] P. Rutgeerts, W. J. Sandborn, B. G. Feagan et al., "Infliximab for induction and maintenance therapy for ulcerative colitis," New England Journal of Medicine, vol. 353, no. 23, pp. 24622476, 2005.

[12] W. J. Sandborn, B. G. Feagan, C. Marano et al., "Subcutaneous golimumab induces clinical response and remission in patients with moderate-to-severe ulcerative colitis," Gastroenterology, vol. 146, no. 1, pp. 85-95, 2014.

[13] M. Barreiro-de Acosta, N. Vallejo, D. de la Iglesia et al., "Evaluation of the risk of relapse in ulcerative colitis according to the degree of mucosal healing (mayo 0 vs 1): a longitudinal cohort study," Journal of Crohn's and Colitis, vol. 10, no. 1, pp. 13-19, 2016.

[14] M. Okada, H. Sakamoto, T. Takezawa et al., "Laterally spreading tumor of the rectum delineated with linked color imaging technology," Clinical Endoscopy, vol. 49, no. 2, pp. 207-208, 2016.

[15] N. Yoshida, Y. Naito, R. Yasuda et al., "Linked color imaging improves the visibility of various featured colorectal polyps in an endoscopist's visibility and color difference value," International Journal of Colorectal Disease, vol. 32, no. 9, pp. 12531260, 2017.

[16] N. Yoshida, Y. Naito, T. Murakami et al., "Linked color imaging improves the visibility of colorectal polyps: a video study," Endoscopy International Open, vol. 5, no. 6, pp. E518-E525, 2017.

[17] T. Suzuki, T. Hara, Y. Kitagawa et al., "Linked-color imaging improves endoscopic visibility of colorectal nongranular flat lesions," Gastrointestinal Endoscopy, vol. 86, no. 4, pp. 692697, 2017.

[18] D. Fujimoto, N. Muguruma, K. Okamoto et al., "Linked color imaging enhances endoscopic detection of sessile serrated adenoma/polyps," Endoscopy International Open, vol. 6, no. 3, pp. E322-E334, 2018.

[19] C. E. Oliveira Dos Santos, D. Malaman, J. C. Pereira-Lima, F. de Quadros Onófrio, and J. M. Ribas Filho, "Impact of linked-color imaging on colorectal adenoma detection," Gastrointestinal Endoscopy, vol. 90, no. 5, pp. 826-834, 2019.

[20] K. Uchiyama, T. Takagi, S. Kashiwagi et al., "Assessment of endoscopic mucosal healing of ulcerative colitis using linked colour imaging, a novel endoscopic enhancement system," Journal of Crohn's and Colitis, vol. 11, no. 8, pp. 963-969, 2017.

[21] S. Kanmura, H. Hamamoto, A. Tanaka et al., "Diagnostic utility of linked color imaging in the evaluation of colonic mucosal inflammation in ulcerative colitis: a pilot study," Endoscopy International Open, vol. 7, no. 8, pp. E937-E943, 2019.

[22] W. Reinisch, W. J. Sandborn, D. W. Hommes et al., “Adalimumab for induction of clinical remission in moderately to severely active ulcerative colitis: results of a randomised controlled trial," Gut, vol. 60, no. 6, pp. 780-787, 2011.

[23] K. Yokoyama, K. Kobayashi, M. Mukae, M. Sada, and W. Koizumi, "Clinical study of the relation between mucosal healing and long-term outcomes in ulcerative colitis," Gastroenterology Research and Practice, vol. 2013, Article ID 192794, 6 pages, 2013.

[24] S. R. Fernandes, J. S. L. D. Pinto, P. Marques da Costa, L. Correia, and GEDII, "Disagreement among gastroenterologists using the mayo and rutgeerts endoscopic scores," Inflammatory Bowel Diseases, vol. 24, no. 2, pp. 254-260, 2018.

[25] Y. Maeda, K. Ohtsuka, S. E. Kudo et al., "Endocytoscopic narrow-band imaging efficiency for evaluation of inflammatory activity in ulcerative colitis," World Journal of Gastroenterology, vol. 21, no. 7, pp. 2108-2115, 2015.

[26] S. Sasanuma, K. Ohtsuka, S. E. Kudo et al., "Narrow band imaging efficiency in evaluation of mucosal healing/relapse of ulcerative colitis," Endoscopy International Open, vol. 6, no. 5, pp. E518-E523, 2018.

[27] T. Guo, J.-M. Qian, A.-M. Yang, Y. Li, and W.-X. Zhou, "Narrow-band imaging colonoscopy to assess mucosal angiogenesis in ulcerative colitis," Gastroenterology Research and Practice, vol. 2019, Article ID 8312624, 7 pages, 2019.

[28] T. Inoue, M. Murano, N. Murano et al., "Comparative study of conventional colonoscopy and pan-colonic narrow-band imaging system in the detection of neoplastic colonic polyps: a randomized, controlled trial," Journal of Gastroenterology, vol. 43, no. 1, pp. 45-50, 2008.

[29] A. Adler, J. Aschenbeck, T. Yenerim et al., "Narrow-band versus white-light high definition television endoscopic imaging for screening colonoscopy: a prospective randomized trial," Gastroenterology, vol. 136, no. 2, pp. 410-416.e1, 2009.

[30] R. Bisschops, T. Bessissow, J. A. Joseph et al., "Chromoendoscopy versus narrow band imaging in UC: a prospective randomised controlled trial," Gut, vol. 67, no. 6, pp. 1087-1094, 2018.

[31] H. Ikematsu, T. Sakamoto, K. Togashi et al., "Detectability of colorectal neoplastic lesions using a novel endoscopic system with blue laser imaging: a multicenter randomized controlled trial," Gastrointestinal Endoscopy, vol. 86, no. 2, pp. 386-394, 2017. 